\title{
Research Article Effects of Cable on the Dynamics of a Cantilever Beam with Tip Mass
}

\author{
Yi-Xin Huang, Hao Tian, and Yang Zhao \\ School of Astronautics, Harbin Institute of Technology, Harbin 150001, China \\ Correspondence should be addressed to Yang Zhao; yangzhao@hit.edu.cn
}

Received 15 August 2016; Accepted 9 October 2016

Academic Editor: Salvatore Russo

Copyright (c) 2016 Yi-Xin Huang et al. This is an open access article distributed under the Creative Commons Attribution License, which permits unrestricted use, distribution, and reproduction in any medium, provided the original work is properly cited.

\begin{abstract}
The dynamic effects of cable attachment on a cantilever beam with tip mass are investigated by an improved Chebyshev spectral element method. The cabled beam is modeled as a double-beam system connected by springs at several discrete locations. By utilizing high order Chebyshev polynomials as basis functions and meshing the system at the locations of connections, precise numerical results of the natural frequencies and mode shapes can be obtained using only a few elements. The accuracy of this method is validated through comparing the results of finite element method and those of spectral element method in literature. The validated method is implemented to investigate the effects of parameters, including spring stiffness, number of connections, density, and Young's modulus of cable. The results show that the mode shapes of the cabled beam system can be classified into two types: beam mode shapes and cable mode shapes, according to their main deformation. Their corresponding natural frequencies change in very different ways with the variation of system parameters. This work can be applied to optimize the dynamic characteristics of precise spacecraft structures with cable attachments.
\end{abstract}

\section{Introduction}

In spacecraft applications, modeling and evaluating dynamic behavior accurately during launch and in orbit are extremely important. Cables as power and signal transfers are used in almost all satellites. Previous tests have shown that cables have remarkable influences on precision space structures, not only changing the mass distribution but also adding damping and in general changing the entire dynamic response $[1,2]$. The influence of cables on space structures as a structural element is a growing but relatively new concern, and as such, there are only a few published studies that investigate modeling space cables specifically [3].

Currently, the most commonly used method for modeling cabled structure is nonstructural mass method, including lumped mass method which adds lumped mass at attachment locations and distributed mass method which adds cable as a distributed mass by changing the density of the host structure. But both of these two methods have been proved insufficient to modeling precise cabled structures connected at discrete locations because they ignore the dynamic interaction between the cable and its host structure $[4,5]$. In space mission, an imprecise dynamic model might degrade mission performance and destabilize structural control loops [6]. In order to improve modeling precision, the double-beam system was proposed for modeling cabled beams, in which cable is modeled as a beam to contain its bending stiffness and shear deformation, and springs are used to represent discrete connections [7]. Although the dynamic characteristics of a single beam can be obtained exactly by many ways, when a secondary beam is attached to the main beam by springs at several places, its solution becomes complicated. Previous studies mostly focus on beams with continuous elastica connection such as sandwich beam and composite beam $[8,9]$, or beams with string connected at their ends [10]. Only a few researchers have performed dynamic analysis for the double-beam system connected by springs at several discrete locations. Babuška et al. [11] established a finite element model for a cabled beam using software Nastran and got its frequency response functions. But the preprocess of finite element method (FEM) is usually tedious, especially when the number or location of connections is changed. Spak et al. developed a method to calculate cable properties that are suitable to use for modeling purpose from direct 
physical measurements of cables [3, 12], compared different damping models for space flight cables [13], and used the distributed transfer function method to model cables and a simple cabled structure [5]. Choi $[14,15]$ studied the natural frequencies and damping of a cabled-harnessed structure using spectral element method (SEM). The effects of the number of connections, the spring stiffness of interconnections, and the mass portion of an attached cable had also been analyzed. However, this method is actually a semianalytical and seminumerical method, needing to resolve analytical solution of dispersion relations for every type of model. Besides, because the SEM is a time-domain method, it is inconvenient to obtain the mode shapes of cabled structures. Remedia et al. [16] investigated the influence of harness on spacecraft honeycomb panel through an experimental campaign. Zhang et al. [17] presented a general analytical approach for studying transverse vibrations of double-beam systems, made of two Timoshenko beams connected by discrete springs and coupled with multiple discontinuities.

However, mostly previous researches focus on beams with free-free boundary, and the changes of mode shapes due to attached cables are also rarely discussed. Cantilever beam with a tip mass as shown in Figure 1 is a typical model for many beam-like space structures equipped with instrument at its tip. Cables are usually essential for signal and power transmission between the hub and tip equipment. If the tip equipment is a very sophisticated machine and has a high request to the dynamics characteristics, the effects of the attached cable must be taken into account. This is just the goal of this research. To that end, the effects of cables property parameters, such as density and Young's modulus, need to be investigated and the influences of connections' number, stiffness, and locations also should be studied. In order to get the precise numerical solutions of both natural frequencies and mode shapes, our approach used here is a Chebyshev spectral element method (CSEM) improved from spectralTchebychev technique which was developed by Yagci for solving linear and nonlinear beam equations $[18,19]$. This technique uses orthogonal Chebyshev polynomials as basis functions and applies Galerkin's method to obtain the solution. It has high accuracy and exponential convergence, and only a small number of polynomials are sufficient to obtain the machine-precision accuracy. It also requires fewer elements than the FEM approach, not needing remeshing after the connections' locations changed. Using projection matrices, any linear boundary conditions can be incorporated. Different from the meaning of SEM in literature [14] where Fourier spectral decomposition is used for time-domain analysis, "spectral" in CSEM means to perform spatial discretization in elements using Chebyshev spectral method.

\section{Problem Description}

Consider a cantilever beam with tip mass and cable attachment in Figure 1 which can be modeled as a double-beam system as shown in Figure 2. Both the host beam and the cable attachment are considered as Timoshenko beams to include the effects of shear deformation and rotatory inertia. They are connected by translational and torsional springs at several

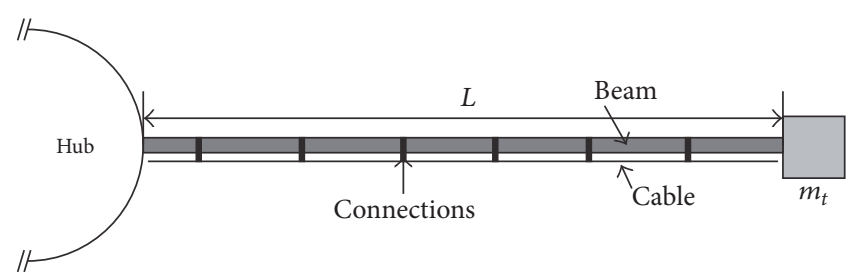

Figure 1: A cantilever beam with tip mass and cable attachment.

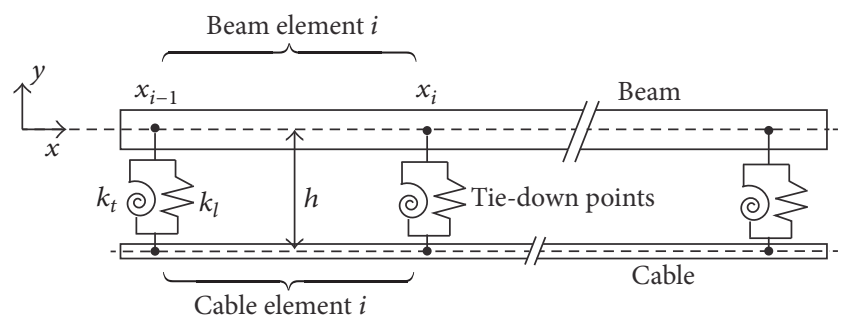

FIGURE 2: Double-beam model for a cabled beam.

discrete points; $k_{t}$ and $k_{l}$ are the translational and torsional stiffness, respectively. The rotational inertia of tip mass is ignored. Because the two ends of the cable are usually slack, free-free boundary conditions are adopted. The excitation levels are thought to be in the linear region. Nonlinear behavior, such as slack cables slapping against the host beam, is not considered here. Based on this model, the Chebyshev spectral element model will be established for this cabled beam system, and numerical results of natural frequencies and mode shapes will be compared with the results of FEM and SEM. Then this validated model will be used to investigate the changes of dynamic characteristics of a cabled beam with variation of cable parameters, such as density and Young's modulus. The influences of the connection number and springs' stiffness will also be discussed.

\section{Chebyshev Spectral Element Method}

The discrete connections divide the beam and the cable into several parts. The CSEM used herein meshes the beam and the cable in the places of their connections and then assemblies them like FEM. It is assumed that there are $n-1$ connections, $n$ cable elements, and $n$ beam elements. Adding the tip mass element, there are totally $2 n+1$ elements. But unlike FEM which use low order polynomials in "small" elements, this method uses high order Chebyshev polynomials to approximate the deformation in "big" elements. The main advantage of the CSEM is that no remeshing is needed after the locations or number of the connections changed. Besides, only needing to change the number of polynomials, a good balance between accuracy and efficiency can be obtained. It can be considered as a combination of the Chebyshev spectral method (CSM) and the finite element method, combining the advantages of both methods. Furthermore, in order to deal with different boundary conditions in the same way, a projection matrix method will be used. In this section, we firstly review the properties of the Chebyshev polynomials 
and then give the Chebyshev spectral element model of this double-beam system.

3.1. Chebyshev Polynomials. The Chebyshev polynomials are a group of recursive orthogonal polynomials which can be given by [18]

$$
T_{k}(x)=\cos (k \arccos (x))
$$

where $k=1,2, \ldots$ and $x \in[-1,1]$. For an arbitrary interval $x \in\left[l_{1}, l_{2}\right]$, a mapping

$$
\begin{aligned}
& x(\xi)=\xi \frac{l_{2}-l_{1}}{2}+\frac{l_{2}+l_{1}}{2}, \\
& \xi(x)=\frac{2}{l_{2}-l_{1}} x-\frac{l_{2}+l_{1}}{l_{2}-l_{1}}
\end{aligned}
$$

can be defined, and then scaled Chebyshev polynomials $T_{k}(\xi(x))$ will be used. The Chebyshev polynomials form a complete set, which means any square-integrable function $y(x)$ which is infinitely differentiable in the interval $[0,1]$ can be approximated by a truncated Chebyshev series expansion as

$$
y(x, t)=\sum_{k=0}^{N-1} a_{k}(t) T_{k}(x)
$$

where $N$ is the number of polynomials used for the truncated expansion.

Adopting Gauss-Lobatto sampling method,

$$
x_{k}=\cos \left(\frac{k \pi}{(N-1)}\right), \quad k=0,1,2, \ldots, N-1 .
$$

The values of function $y(x, t)$ at spatial sampling points $x_{k}$ form a vector $\mathbf{y}=\left\{y_{k}\right\}$. There exists a one-to-one mapping between the expansion coefficients vector $\mathbf{a}=\left\{a_{k}\right\}$ and sampling value vector $\mathbf{y}$ :

$$
\begin{aligned}
& \mathbf{a}=\mathbf{T}_{F} \mathbf{y}, \\
& \mathbf{y}=\mathbf{T}_{B} \mathbf{a},
\end{aligned}
$$

where $\mathbf{T}_{F}$ is an $N \times N$ forward transformation matrix and $\mathbf{T}_{B}$ is the inverse matrix of $\mathbf{T}_{F}$.

The derivative of each Chebyshev polynomial can be recursively expressed in terms of lower order Chebyshev polynomials. Therefore, the approximation of the spatial derivative $y^{\prime}$ can be written as

$$
y^{\prime}(x, t)=\sum_{k=0}^{N-1} b_{k}(t) T_{k}(x)
$$

where coefficient vector $\mathbf{b}=\left\{b_{k}\right\}$ can be got from a through a different matrix $\mathbf{D}$ which just relates to the number of Chebyshev polynomials and the definition domain of $y$.

$$
\mathbf{b}=\mathbf{D} \mathbf{a} .
$$

Similarly, the expansion coefficient vector $\mathbf{a}^{(n)}$ of the $n$th spatial derivative of $y$ can be given as

$$
\mathbf{a}^{(n)}=\mathbf{D}^{n} \mathbf{a}
$$

and then the $n$th spatial derivative of $y$ can be obtained as

$$
\mathbf{y}^{(n)}=\mathbf{T}_{B} \mathbf{a}^{(n)}=\mathbf{T}_{B} \mathbf{D}^{n} \mathbf{T}_{F} \mathbf{y}=\mathbf{Q}_{n} \mathbf{y},
$$

where $\mathbf{Q}_{n}$ is called the $n$th derivative matrix.

The definite integral of $y$ can be described as

$$
\int_{l_{1}}^{l_{2}} y d x=\mathbf{v}^{T} \mathbf{a}
$$

where $\mathbf{v}$ is the definite integral vector.

The inner product of functions $f(x)$ and $g(x)$ can also be expressed by Chebyshev polynomials as follows:

$$
\int_{l_{1}}^{l_{2}} f(x) g(x) d x=\mathbf{f}^{T} \mathbf{V g},
$$

where $\mathbf{V}$ is the inner-product matrix.

3.2. Timoshenko Beam Element. The governing equation of a uniform Timoshenko beam is represented by [13]

$$
\begin{aligned}
\rho A \ddot{y}+c_{y} \dot{y}-\kappa G A\left(y^{\prime \prime}-\varphi^{\prime}\right) & =f \\
\rho I \ddot{\varphi}+c_{\varphi} \dot{\varphi}-E I \varphi^{\prime \prime}-\kappa G A\left(y^{\prime}-\varphi\right) & =m,
\end{aligned}
$$

where $y$ and $\varphi$ are the transverse displacement and the slope due to bending, respectively. $\rho$ is the material density, $A$ is the cross-sectional area, $G$ is the shear modulus, $E$ is Young's modulus, and $I$ is the second moment of inertia. Damping for the displacement and rotation is included through coefficients $c_{y}$ and $c_{\varphi} . f$ and $m$ are the applied force and moment, respectively.

Assume that the approximate solution of (12) in the spectral form is

$$
\begin{aligned}
& y(x, t) \approx \sum_{k=0}^{N-1} a_{y k}(t) T_{k}(\xi(x)) \\
& \varphi(x, t) \approx \sum_{k=0}^{N-1} a_{\varphi k}(t) T_{k}(\xi(x)) .
\end{aligned}
$$

Here, Chebyshev polynomials are chosen as trial functions. Due to the fact that (13) is an approximation, submitting (13) into (12) will produce an error or residual. According to Galerkin's method, the weighted residuals must be minimized; namely,

$$
\begin{aligned}
& \int_{0}^{L} \theta_{y}\left(\rho A \ddot{y}+c_{y} \dot{y}-\kappa G A\left(y^{\prime \prime}-\varphi^{\prime}\right)-f\right) d x=0 \\
& \int_{0}^{L} \theta_{\varphi}\left(\rho I \ddot{\varphi}+c_{\varphi} \dot{\varphi}-E I \varphi^{\prime \prime}-\kappa G A\left(y^{\prime}-\varphi\right)-m\right) d x \\
& \quad=0,
\end{aligned}
$$


where $\theta_{y}$ and $\theta_{\varphi}$ are the weighting functions coinciding with the trial functions. Using the inner-product expression from (11), the residuals can be rewritten as

$$
\begin{aligned}
& \boldsymbol{\theta}_{y}^{T} \mathbf{V}\left[\rho A \ddot{\mathbf{y}}+c_{y} \dot{\mathbf{y}}-\kappa G A\left(\mathbf{Q}_{2} \mathbf{y}-\mathbf{Q}_{1} \boldsymbol{\varphi}\right)-\mathbf{f}\right]=0 \\
& \boldsymbol{\theta}_{\varphi}^{T} \mathbf{V}\left[\rho I \ddot{\boldsymbol{\varphi}}+c_{\varphi} \dot{\boldsymbol{\varphi}}-E I \mathbf{Q}_{2} \boldsymbol{\varphi}-\kappa G A\left(\mathbf{Q}_{1} \mathbf{y}-\boldsymbol{\varphi}\right)-\mathbf{m}\right] \\
& \quad=0 .
\end{aligned}
$$

Since this equation should be satisfied for arbitrary $\theta_{y}$ and $\theta_{\varphi}$, there must be

$$
\begin{array}{r}
\mathbf{V}\left[\rho A \ddot{\mathbf{y}}+c_{y} \dot{\mathbf{y}}-\kappa G A\left(\mathbf{Q}_{2} \mathbf{y}-\mathbf{Q}_{1} \boldsymbol{\varphi}\right)-\mathbf{f}\right]=\mathbf{0} \\
\mathbf{V}\left[\rho I \ddot{\boldsymbol{\varphi}}+c_{\varphi} \dot{\boldsymbol{\varphi}}-E I \mathbf{Q}_{2} \boldsymbol{\varphi}-\kappa G A\left(\mathbf{Q}_{1} \mathbf{y}-\boldsymbol{\varphi}\right)-\mathbf{m}\right]=\mathbf{0}
\end{array}
$$

and this equation can be expressed in matrix form as

$$
\mathbf{M}_{s} \ddot{\mathbf{q}}+\mathbf{C}_{s} \dot{\mathbf{q}}+\mathbf{K}_{s} \mathbf{q}-\mathbf{F}_{s}=\mathbf{0},
$$

where

$$
\begin{aligned}
& \mathbf{M}_{s}=\mathbf{V}_{s} \mathbf{M} \text {, } \\
& \mathbf{C}_{s}=\mathbf{V}_{s} \mathbf{C} \text {, } \\
& \mathbf{K}_{s}=\mathbf{V}_{s} \mathbf{K} \text {, } \\
& \mathbf{F}_{s}=\mathbf{V}_{s} \mathbf{F} \text {, } \\
& \mathbf{V}_{s}=\left[\begin{array}{ll}
\mathbf{V} & 0 \\
\mathbf{0} & \mathbf{V}
\end{array}\right], \\
& \mathbf{q}=\left[\begin{array}{l}
\mathbf{y} \\
\varphi
\end{array}\right], \\
& \mathbf{M}=\left[\begin{array}{cc}
\rho A \mathbf{I} & \mathbf{0} \\
\mathbf{0} & \rho I \mathbf{I}
\end{array}\right], \\
& \mathbf{C}=\left[\begin{array}{cc}
c_{y} \mathbf{I} & \mathbf{0} \\
\mathbf{0} & c_{\varphi} \mathbf{I}
\end{array}\right] \\
& \mathbf{K}=\left[\begin{array}{cc}
-\kappa G A \mathbf{Q}_{2} & \kappa G A \mathbf{Q}_{1} \\
-\kappa G A \mathbf{Q}_{1} & \kappa G A \mathbf{I}-E I \mathbf{Q}_{2}
\end{array}\right] \text {, } \\
& \mathbf{F}=\left[\begin{array}{l}
\mathbf{f} \\
\mathbf{m}
\end{array}\right] \text {. }
\end{aligned}
$$

Here, $\mathbf{q}$ is the $2 N$ element displacement vector, $\mathbf{I}$ is a $N \times$ $N$ identity matrix, and $\mathbf{Q}_{i}$ is the $i$ th Chebyshev derivative matrix defined in (9). This is the discrete equation of motion of a Timoshenko viscous beam without imposing boundary conditions.

3.3. Beam-Cable Coupled System. Now, consider the doublebeam system in Figure 2 which has $2 n+1$ elements. For the tip mass, at $x=L$ there are

$$
\begin{aligned}
m_{t} \ddot{y}_{t}-\kappa G A\left(y_{B}^{\prime}-\varphi_{B}\right) & =0, \\
y_{t} & =y_{B} .
\end{aligned}
$$

Define

$$
\begin{aligned}
& \mathbf{q}_{G}{ }^{T}=\left\{\begin{array}{lllllll}
\mathbf{q}_{B, 1}{ }^{T} & \cdots & \mathbf{q}_{B, n}{ }^{T} & \mathbf{q}_{C, 1}{ }^{T} & \cdots & \mathbf{q}_{C, n}{ }^{T} & y_{t}
\end{array}\right\} \\
& \mathbf{M}_{G}=\left[\begin{array}{ccc}
\mathbf{M}_{B, i} & \mathbf{0} & \mathbf{0} \\
\mathbf{0} & \mathbf{M}_{C, i} & \mathbf{0} \\
\mathbf{0} & \mathbf{0} & m_{t}
\end{array}\right] \text {, } \\
& \mathbf{C}_{G}=\left[\begin{array}{ccc}
\mathbf{C}_{B, i} & \mathbf{0} & \mathbf{0} \\
\mathbf{0} & \mathbf{C}_{C, i} & \mathbf{0} \\
\mathbf{0} & \mathbf{0} & 0
\end{array}\right] \\
& \mathbf{K}_{G}=\left[\begin{array}{ccc}
\mathbf{K}_{B, i} & \mathbf{0}_{2 n N \times 2 n N} & \mathbf{0}_{2 n N \times 1} \\
\mathbf{0}_{2 n N \times 2 n N} & \mathbf{K}_{C, i} & \mathbf{0}_{2 n N \times 1} \\
\mathbf{K}_{31} & \mathbf{0}_{1 \times 2 n N} & 0
\end{array}\right] \\
& \mathbf{K}_{31}=\left[\begin{array}{lll}
\mathbf{0}_{1 \times 2 N(n-1)} & -\kappa G A \mathbf{Q}_{1} \mathbf{e}_{1}^{T} & \kappa G A \mathbf{e}_{1}^{T}
\end{array}\right],
\end{aligned}
$$

where subscripts $B$ and $C$ represent beam and cable, respectively; $i=1,2, \ldots, n ; \mathbf{q}_{G}$ is the $4 n N+1$ global displacement vector, whose components $\mathbf{q}_{B, i}$ and $\mathbf{q}_{C, i}$ are elements' displacement vectors defined in (19); $\mathbf{M}_{G}, \mathbf{C}_{G}$, and $\mathbf{K}_{G}$ are $(4 n N+1) \times(4 n N+1)$ matrices; $\mathbf{M}_{B, i}, \mathbf{C}_{B, i}, \mathbf{K}_{B, i}, \mathbf{M}_{C, i}, \mathbf{C}_{C, i}$, and $\mathbf{K}_{C, i}$ are $2 \mathrm{~N} \times 2 \mathrm{~N}$ matrices defined in (20) and (21), and $i=1,2, \ldots, n . \mathbf{e}_{j}$ is a $1 \times N$ column vector whose $j$ th element is 1 and all other elements are zeros. $\mathbf{0}$ are matrix block with all 0 elements. For clarity, in (25), the number of rows and columns is indicated by subscript.

Considering the relation between spring forces and element displacements at $x_{i}$ in Figure 2, the forces and moments acting on elements are

$$
\begin{gathered}
f_{B i}=-k_{l}\left(y_{B i}-y_{C i}\right), \\
m_{B i}=-k_{t}\left(\varphi_{B i}-\varphi_{C i}\right), \\
f_{C i}=k_{l}\left(y_{B i}-y_{C i}\right), \\
m_{C i}=k_{t}\left(\varphi_{B i}-\varphi_{C i}\right) .
\end{gathered}
$$

Notice that all these forces are functions of displacements at connection locations. So, the global force vector due to spring connections can be written in terms of global displacement vector as

$$
\mathbf{F}_{G}=\mathbf{K}_{S} \mathbf{q}_{G},
$$

where $\mathbf{K}_{S}$ is a diagonal matrix whose $(N(i-1)+1)$ th and $(N i)$ th entries are -1 and $(2 n N+N(i-1)+1)$ and $(2 n N+$ $N i$ )th entries are $1, i=1,2, \ldots, n$. According to (17), we can assembly the global equation and get

$$
\mathbf{M}_{G} \ddot{\mathbf{q}}_{G}+\mathbf{C}_{G} \dot{\mathbf{q}}_{G}+\left(\mathbf{K}_{G}-\mathbf{K}_{S}\right) \mathbf{q}_{G}=\mathbf{0} .
$$

This is the global discrete equation of the beam-cable coupled system without considering element compatibility and boundary conditions. 
3.4. Compatibility and Boundary Conditions. Adjacent elements $(i)$ and $(i+1)$ in Figure 2 should meet displacement compatibility. That is, at the common points $x=x_{i}$, the two sides are required to have equal displacements, slopes, forces, and moments. Mathematically, these compatibility conditions can be expressed as

$$
\begin{array}{r}
y_{B, i}-\left.y_{B, i+1}\right|_{x=x_{i}}=0 \\
\varphi_{B, i}-\left.\varphi_{B, i+1}\right|_{x=x_{i}}=0 \\
\varphi_{B, i}^{\prime}-\left.\varphi_{B, i+1}^{\prime}\right|_{x=x_{i}}=0 \\
\left(y_{B, i}^{\prime}-\varphi_{B, i}\right)-\left.\left(y_{B, i+1}^{\prime}-\varphi_{B, i+1}\right)\right|_{x=x_{i}}=0 .
\end{array}
$$

Considering (9), their discrete form can be written as

$$
\begin{array}{r}
\mathbf{e}_{1}^{T} \mathbf{y}_{B, i}-\mathbf{e}_{N}^{T} \mathbf{y}_{B, i+1}=0 \\
\mathbf{e}_{1}^{T} \boldsymbol{\varphi}_{B, i}-\mathbf{e}_{N}^{T} \boldsymbol{\varphi}_{B, i+1}=0 \\
\mathbf{e}_{1}^{T} \mathbf{Q}_{1} \boldsymbol{\varphi}_{B, i}-\mathbf{e}_{N}^{T} \mathbf{Q}_{1} \boldsymbol{\varphi}_{B, i+1}=0 \\
\left(\mathbf{e}_{1}^{T} \mathbf{Q}_{1} \mathbf{y}_{B, i}-\mathbf{e}_{1}^{T} \boldsymbol{\varphi}_{B, i}\right)-\left(\mathbf{e}_{N}^{T} \mathbf{Q}_{1} \mathbf{y}_{B, i+1}-\mathbf{e}_{N}^{T} \boldsymbol{\varphi}_{B, i+1}\right)=0 .
\end{array}
$$

Besides, when spring stiffness is big enough, these connections in Figure 2 can be thought as rigid constraints. Instead of using global force vector $\mathbf{F}_{s}$ in (28), we can handle them like compatibility conditions in (30). For instance, when linear spring stiffness is big enough, we can get

$$
\mathbf{e}_{1}^{T} \mathbf{y}_{B, i}=\mathbf{e}_{1}^{T} \mathbf{y}_{C, i}, \quad i=1,2, \ldots, n-1 .
$$

Similarly, generic linear boundary conditions can be written as

$$
\sum_{i=1}^{n} \sum_{k=0}^{1} \alpha_{i k j} y_{i}^{(k)}+\sum_{i=1}^{n} \sum_{k=0}^{1} \beta_{i k j} \varphi_{i}^{(k)}=0,
$$

where $k$ is the order of derivative, $i$ indicates $i$ th element, and $j$ denotes the particular boundary (right or left boundary of the element). Its discrete is

$$
\sum_{i=1}^{n} \sum_{k=0}^{1} \alpha_{i k j} \mathbf{e}_{j} \mathbf{Q}_{k} \mathbf{y}_{i}+\sum_{i=1}^{n} \sum_{k=0}^{1} \beta_{i k j} \mathbf{e}_{j} \mathbf{Q}_{k} \boldsymbol{\varphi}_{i}=0 .
$$

For example, for free-free boundary condition, there are

$$
\begin{aligned}
y^{\prime}-\varphi & =0, \\
\varphi^{\prime} & =0, \\
& \text { at } x=0, L .
\end{aligned}
$$

Their discrete form can be written as

$$
\begin{array}{r}
\mathbf{e}_{N}^{T} \mathbf{Q}_{1} \mathbf{y}_{B, 1}-\mathbf{e}_{N}^{T} \boldsymbol{\varphi}_{B, 1}=0 \\
\mathbf{e}_{N}^{T} \mathbf{Q}_{1} \boldsymbol{\varphi}_{B, 1}=0 \\
\mathbf{e}_{1}^{T} \mathbf{Q}_{1} \mathbf{y}_{B, n}-\mathbf{e}_{N}^{T} \boldsymbol{\varphi}_{B, n}=0 \\
\mathbf{e}_{1}^{T} \mathbf{Q}_{1} \boldsymbol{\varphi}_{B, n}=0 .
\end{array}
$$

(31), (32), and (36) can be written in terms of global displacement vector as

$$
\mathbf{B q}_{G}=\mathbf{b},
$$

where $\mathbf{B}$ is the compatibility and boundary condition matrix whose rows are determined by (31), (32), and (36) and $\mathbf{b}$ is a column vector whose element is the temporal part of the boundary conditions. Here, $\mathbf{b}$ is a zero vector.

An effective way of imposing the boundary is using projection matrices [19]; in this approach $\mathbf{q}_{G}$ can be expressed as

$$
\mathbf{q}_{G}=\mathbf{P} \widetilde{\mathbf{q}}_{G}+\mathbf{R} \mathbf{b},
$$

where $\mathbf{P}$ and $\mathbf{R}$ are $(4 n N+1) \times(4 n N+1-M)$ and $(4 n N+1) \times M$ projection matrices, respectively, which can be determined using singular value of $\mathbf{B}$. And $\widetilde{\mathbf{q}}_{G}$ is a $(4 n N+1-M)$ vector which satisfies homogeneous boundary conditions, while ensuring $\mathbf{q}_{G}$ satisfies all the boundary conditions. And $M$ is the number of compatibility and boundary conditions.

Substituting (38) into (29) and left-multiplying both sides with $\mathbf{P}^{T}$ result in

$$
\widetilde{\mathbf{M}}_{G} \ddot{\widetilde{\mathbf{q}}}_{G}+\widetilde{\mathbf{C}}_{G} \dot{\tilde{\mathbf{q}}}_{G}+\widetilde{\mathbf{K}}_{G} \widetilde{\mathbf{q}}_{G}=\mathbf{0},
$$

where

$$
\begin{aligned}
\widetilde{\mathbf{M}}_{G} & =\mathbf{P}^{T} \mathbf{M}_{G} \mathbf{P}, \\
\widetilde{\mathbf{C}}_{G} & =\mathbf{P}^{T} \mathbf{C}_{G} \mathbf{P}, \\
\widetilde{\mathbf{K}}_{G} & =\mathbf{P}^{T}\left(\mathbf{K}_{G}-\mathbf{K}_{S}\right) \mathbf{P} .
\end{aligned}
$$

(39) is the discrete governing equation of the cabled beam system. Using state-space method, the numerical results of the natural frequencies and modal shapes can be obtained.

\section{Method Evaluation}

To evaluate the accuracy and convergence of CSEM, two cases were studied, including a double-beam system with free-free boundary and a cantilever cabled beam with tip mass. Their results were compared with the results of SEM in literature [14] and the results of FEM based on Nastran, respectively.

4.1. Free-Free Double-Beams System. Consider the free-free double-beam system in literature [14], whose material properties are shown in Table 1. Using CSEM, the calculated first 6 natural frequencies are summarized in Table 2, where 8 polynomials were used in every element. The results in literature [14] where SEM was used are also included in Table 2. It can be seen that the differences between CSEM and SEM are all less than $0.100 \%$.

Furthermore, the mode shapes can also be obtained using CSEM, as shown in Figures 3 and 4 . The blue lines represent the beam's mode shapes, and the orange lines indicate the cable's mode shapes. Comparing with the mode shapes of a bare beam, it is obvious that the mode shapes can be classified into two categories based on their main 
TABLE 1: Parameters of double beams.

\begin{tabular}{lccccc}
\hline & $L(\mathrm{~m})$ & $A\left(\mathrm{~m}^{2}\right)$ & $I\left(\mathrm{~m}^{4}\right)$ & $E\left(\mathrm{~N} / \mathrm{m}^{2}\right)$ & $\rho\left(\mathrm{kg} / \mathrm{m}^{3}\right)$ \\
\hline Beam 1 & 1.2192 & $8.0645 \times 10^{-5}$ & $6.7746 \times 10^{-11}$ & $7 \times 10^{10}$ & 2700 \\
Beam 2 & 1.2192 & $4.03225 \times 10^{-5}$ & $2.7098 \times 10^{-12}$ & $2.333 \times 10^{10}$ & 540 \\
\hline
\end{tabular}

TABLE 2: Natural frequencies (Hz) of the double-beam system.

\begin{tabular}{lccccccc}
\hline Mode & \multicolumn{3}{c}{$\begin{array}{c}\text { connections } \\
\text { CSEM 8 }\end{array}$} & Error & SEM & $\begin{array}{c}\text { Connections } \\
\text { CSEM 8 }\end{array}$ & Error \\
\hline 1 & SEM & 0.0004 & & 0.0053 & 0 & 0 \\
2 & 6.0119 & 6.8086 & $0.001 \%$ & 10.5753 & 10.5755 & $0.002 \%$ & 11.1792 \\
3 & 6.8085 & 7.1857 & $0.018 \%$ & 15.7602 & 15.7683 & $0.050 \%$ & 30.8144 \\
4 & 7.1844 & 11.1686 & $0.001 \%$ & 16.2981 & 16.3076 & $0.058 \%$ & 60.4046 \\
5 & 11.1687 & 29.5265 & $0.004 \%$ & 30.1176 & 30.1166 & $0.003 \%$ & 99.8429 \\
6 & 29.5254 & 34.6469 & $0.001 \%$ & 58.0927 & 58.0983 & $0.010 \%$ & 149.1315 \\
\hline
\end{tabular}
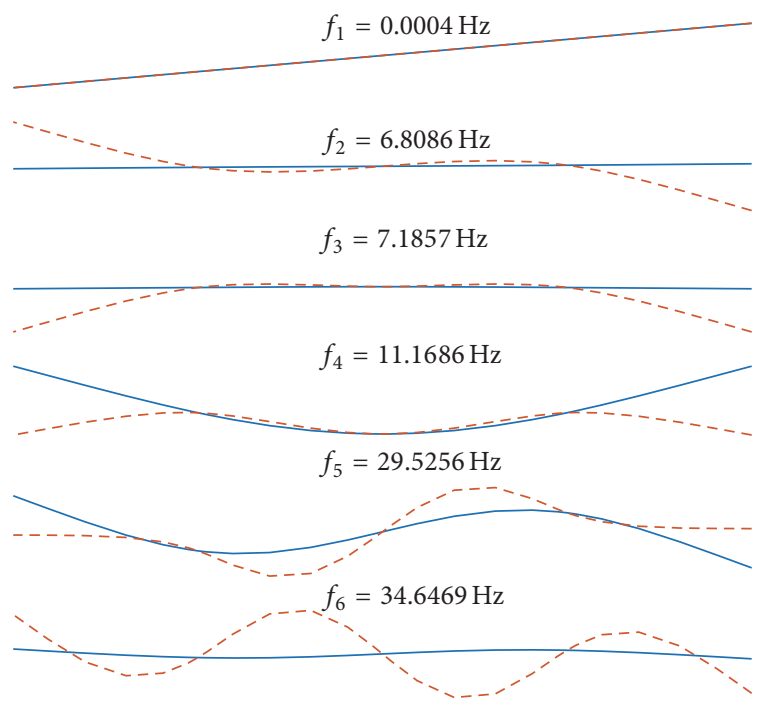

FIGURE 3: Mode shapes of the free-free double-beam system when using 3 connections.

deformation component: mode shapes dominated by beam deformation (beam mode) and mode shapes dominated by cable deformation (cable mode). The corresponding natural frequencies of beam modes are underlined in Table 2. It should be noticed that the first four natural frequencies of the bare beam are $0 \mathrm{~Hz}, 11.1792 \mathrm{~Hz}, 30.8144 \mathrm{~Hz}$, and $60.4046 \mathrm{~Hz}$, respectively. They decrease slightly after being attached to a cable. It is also obvious that when the cable and beam are connected by 3 springs, the first flexible mode is no longer a beam mode. This indicates that the number of connections is a key factor to determine the first flexible mode shape of the cabled beam system.

4.2. Cantilever Beam with Tip Mass and Cable Attachment. Recall the cantilever beam with tip mass and cable attachment in Figure 1. The following parameters were used for the beam: length $L=1 \mathrm{~m}$, radius of cross section $r=0.01 \mathrm{~m}$, Young's

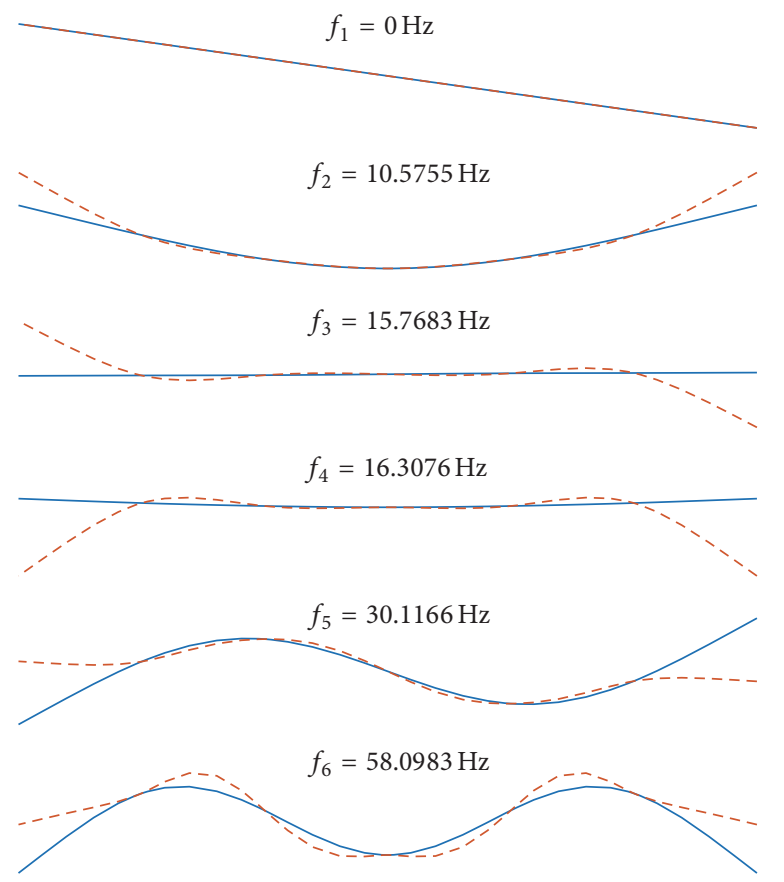

FIGURE 4: Mode shapes of the free-free double-beam system when using 5 connections.

modulus $E=70 \mathrm{GPa}$, material density $\rho=2766.7 \mathrm{~kg} / \mathrm{m}^{3}$, shear factor $\kappa=0.889$, Poisson ratio $v=0.3$, and the tip mass $m_{t}=0.1 \mathrm{~kg}$.

First, taking no account of the cable, we can get the natural frequencies and mode shapes of the cantilever beam with tip mass as shown in Figure 5 utilizing CSEM where 10 Chebyshev polynomials were used totally. Meanwhile, we use the finite element software MSC/NASTRAN to resolve this problem. A lumped point mass element (CONM2) and 50 beam elements (CBAR) were used to represent the tip mass and cantilever beam, respectively. The results we got are $11.626 \mathrm{~Hz}, 76.450 \mathrm{~Hz}$, and $219.85 \mathrm{~Hz}$. Both results agree well, but obviously CSEM is more efficient because it can get a high precise result only using a few polynomials. 

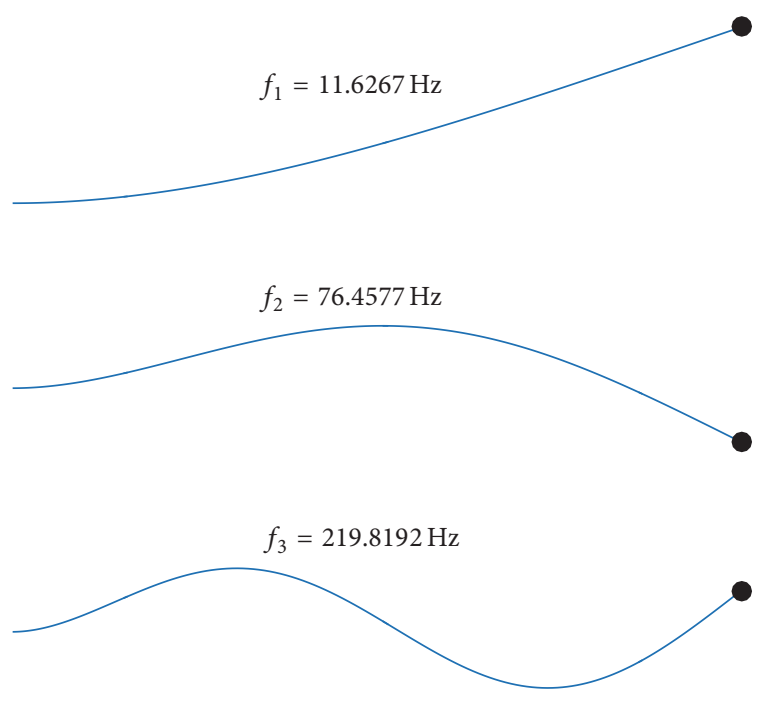

FIGURE 5: Mode shapes of the cantilever beam with tip mass.

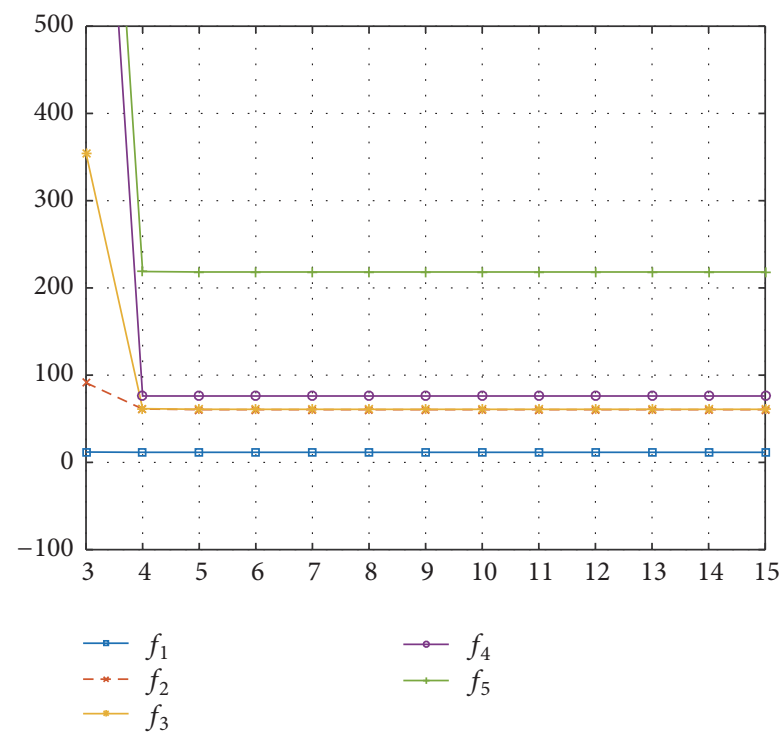

FIGURE 6: The convergence of the first five natural frequencies.

And then, we consider the cable's effects using CSEM. The parameters of the cable used here include length $L_{c}=$ $1 \mathrm{~m}$, radius $r_{c}=0.002 \mathrm{~m}$, density $\rho_{c}=1000 \mathrm{~kg} / \mathrm{m}^{3}$, Young's modulus of $E=20 \mathrm{GPa}$, shear factor $\kappa=0.889$, and Poisson ratio $v=0.3$. The connection parameters include linear spring stiffness $k_{l}=10^{6} \mathrm{~N} / \mathrm{m}$ and torsional spring stiffness $k_{t}=0 \mathrm{~N} \cdot \mathrm{m} / \mathrm{rad}$, and number of connections is 5 ; that is, $n=6$. The convergence of the first five natural frequencies is shown in Figure 6. The results show that, only using 6 polynomials, the relative error $\left(f_{i, N}-f_{i, N-1}\right) / f_{i, N}$ converges to $0.05 \%$. Table 3 lists both the results of FEM using different number of elements and the results of CSEM using different number of polynomials in each element. Figure 7 shows the first five mode shapes. We can see that the results from CSEM are fairly close to those of FEM.
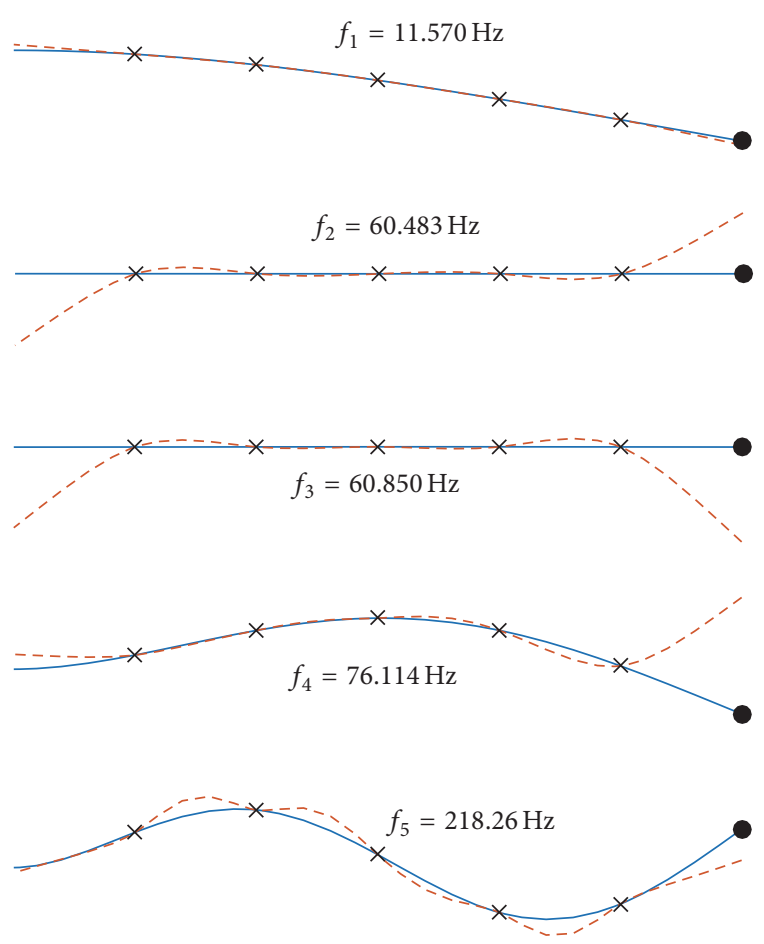

FIGURE 7: Mode shapes of cabled beam with 5 connections when $k_{l}=10^{6} \mathrm{~N} / \mathrm{m}$.

TABLE 3: The natural frequencies $(\mathrm{Hz})$ of the cabled beam with tip mass.

\begin{tabular}{lccccc}
\hline Method & $f_{1}$ & $f_{2}$ & $f_{3}$ & $f_{4}$ & $f_{5}$ \\
\hline FEM 60 & 11.570 & 60.271 & 60.633 & 76.113 & 218.32 \\
FEM 80 & 11.571 & 60.449 & 60.813 & 76.130 & 218.38 \\
FEM 300 & 11.571 & 60.463 & 60.827 & 76.131 & 218.38 \\
CSEM 5 & 11.570 & 60.499 & 60.866 & 76.114 & 218.26 \\
CSEM 6 & 11.570 & 60.484 & 60.851 & 76.114 & 218.26 \\
CSEM 7 & 11.570 & 60.484 & 60.850 & 76.114 & 218.26 \\
CSEM 8 & 11.570 & 60.483 & 60.850 & 76.114 & 218.26 \\
\hline
\end{tabular}

\section{Cable Effects Analysis}

There are many factors that might have influences on the dynamic characteristics of a cabled beam with tip mass. In this section we focus on the effects of connection parameters and cable parameters; both of them might change in a big range.

5.1. Effects of Connection Parameters. Using the same parameters in Section 4.2, the change of natural frequencies with the variation of connection spring stiffness $k_{l}$ is shown in Figure 8 . The first natural frequency is nearly unchanged, and the other ones increase and finally converge to stable values with the increasing of spring stiffness $k_{l}$. When $k_{l}=10^{5} \mathrm{~N} / \mathrm{m}$, all of the first five natural frequencies stabilize. Observe the mode shapes and natural frequencies shown in Figure 7 in which $k_{l}=10^{6} \mathrm{~N} / \mathrm{m}$; it is obvious that the $1 \mathrm{st}$, 4 th, and 5 th modes are beam modes and the 2nd and 3rd modes are cable 


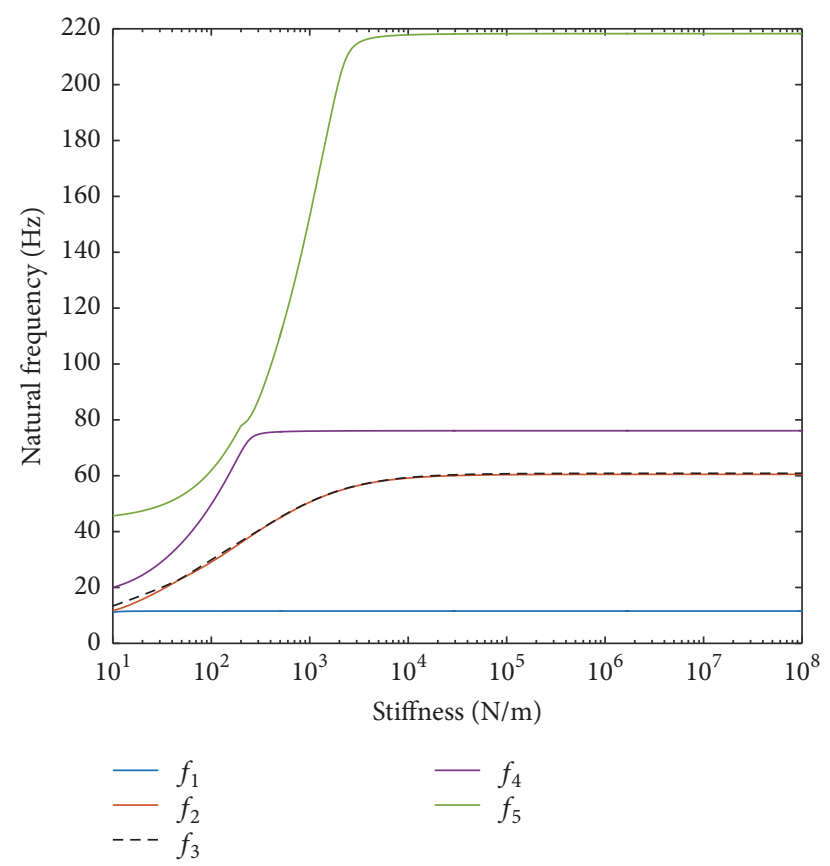

FIGURE 8: Variation of natural frequencies versus connection stiffness $k_{l}$.

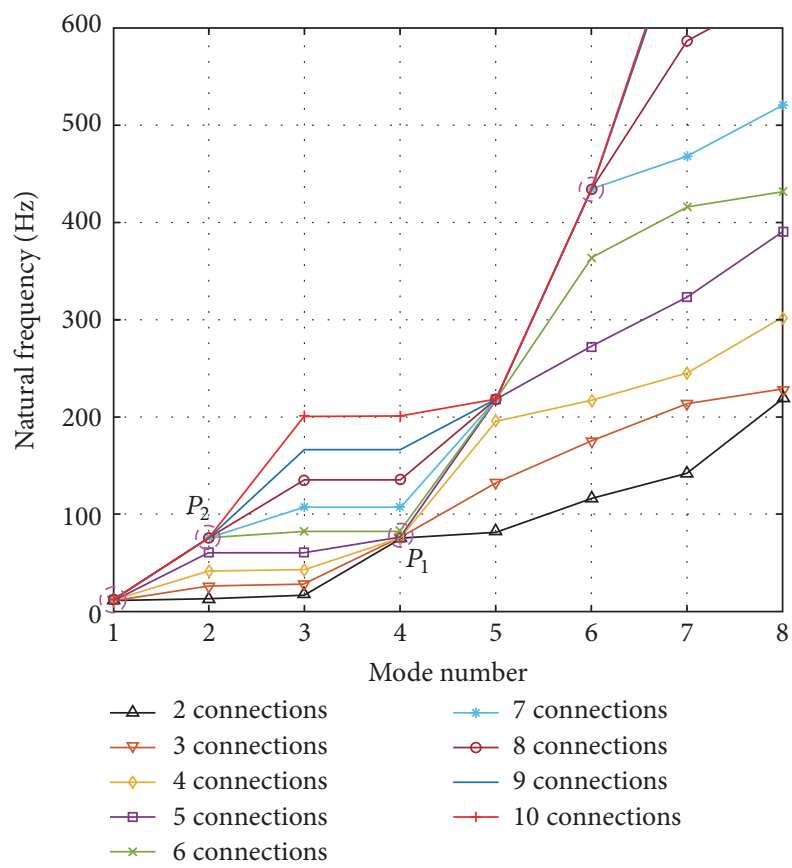

FIGURE 9: Variation of the natural frequencies with the number of connections when $k_{l}=10^{5} \mathrm{~N} / \mathrm{m}$.

modes. So it is concluded that the frequencies of beam mode increase and converge faster than those of beam mode, and the lower frequencies converge faster than higher ones. It also can be seen that the changes of high order frequencies are much bigger than those of low order frequencies.

The change of natural frequencies with the variation of the number of connections is illustrated in Figure 9; here
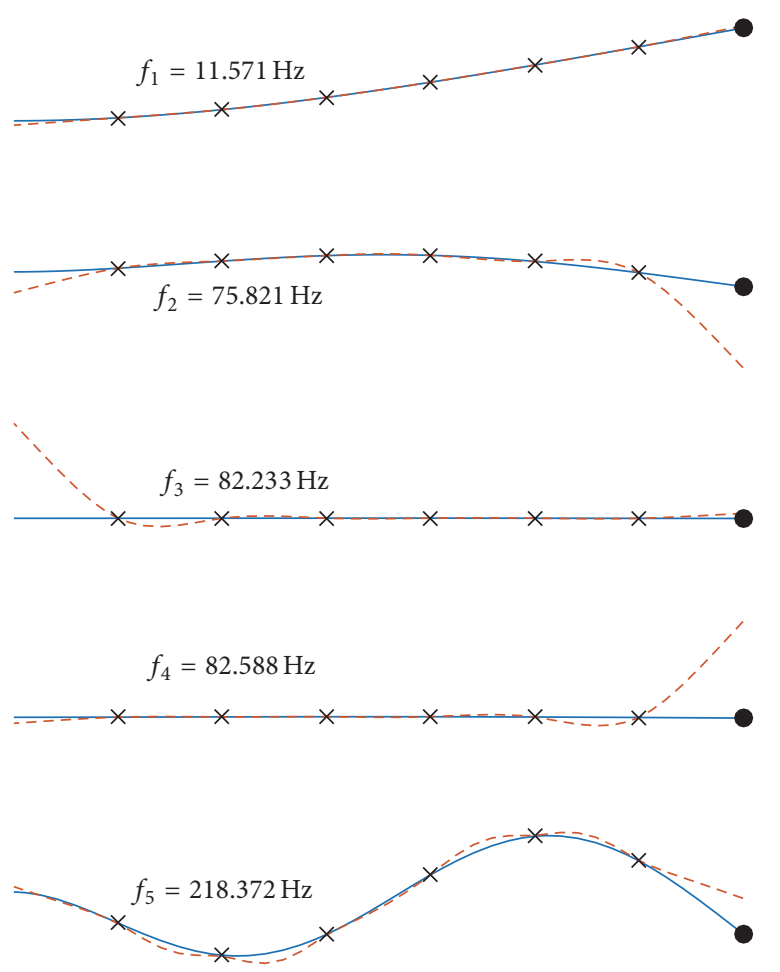

FIgURE 10: Mode shapes of cabled beam with 6 connections when $k_{l}=10^{5} \mathrm{~N} / \mathrm{m}$.

$k_{l}=10^{5} \mathrm{~N} / \mathrm{m}$. As the number of connections increases, the natural frequencies of cable modes increase rapidly and those of beam modes, as shown in purple circle, keep nearly unchanged. This phenomenon results in mode shapes exchanges between adjacent modes. Remember that when the number of connections is 5 , the 1 st, 4 th, and 5 th modes are beam modes. Observe the mode shapes in Figure 10 where the number of connections changed to 6 , the 1st, $2 \mathrm{nd}$, and 5 th modes become beam modes, and 3rd and 4 th modes become cable modes. That is because the natural frequencies of cable modes changed from $60.360 \mathrm{~Hz}$ and $60.705 \mathrm{~Hz}$ to $75.821 \mathrm{~Hz}$ and $82.233 \mathrm{~Hz}$, as shown in figure points $P_{1}$ and $P_{2}$.

5.2. Effects of Cable Parameters. Modeling a spaceflight cable as a homogenous beam requires inputting various equivalent parameters, such as density and Young's modulus. In engineering practice, there are many different kinds of cables with a wide range of material properties. The effects due to the changing of cable parameters, including density and Young's modulus, are discussed in this section.

Figure 11 illustrates the variation of the natural frequencies with the cable's density. It can be observed that the natural frequencies of cable modes decline gradually with the increasing of the cable's density. The low order natural frequencies of beam modes keep nearly unchanged, and high order ones have a slight decline. As a result, adjacent frequency curves will cross at a series of special points, such as points $C_{1}$ and $C_{2}$ in Figure 11. Compare the mode shapes at points $A$ and $B$, where the density, respectively, is $1500 \mathrm{~kg} / \mathrm{m}^{3}$ 


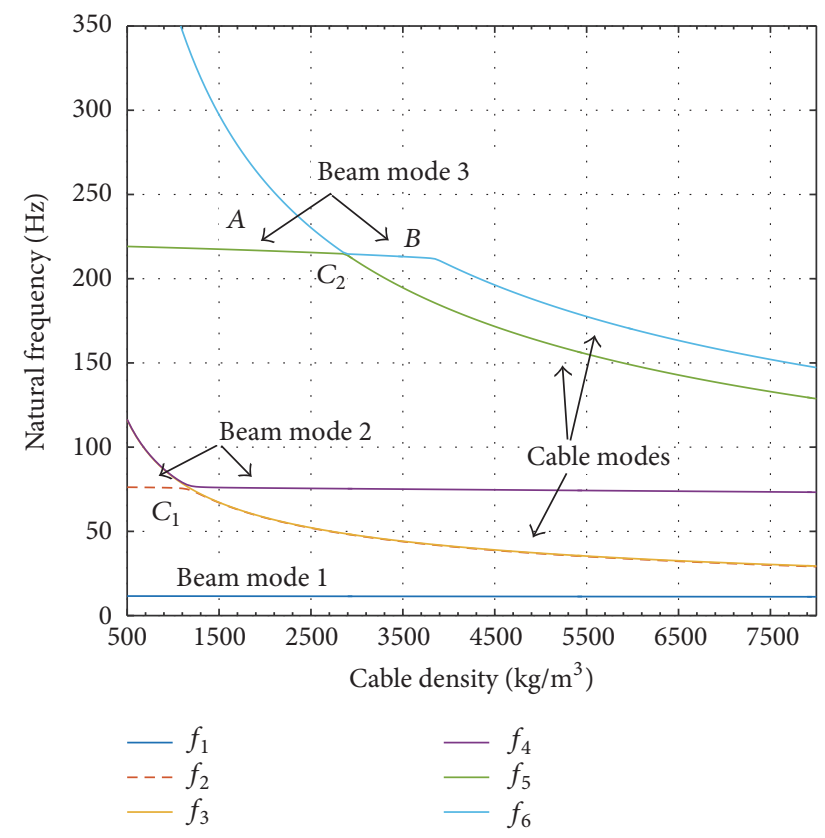

FIGURE 11: Variation of the natural frequencies with the density of cable, 6 connections, and $k_{l}=10^{5} \mathrm{~N} / \mathrm{m}$.

and $3500 \mathrm{~kg} / \mathrm{m}^{3}$, as shown in Figure 12. The natural frequency decreases to $213.12 \mathrm{~Hz}$, and the deformation of the cable increases obviously, due to the increasing of density.

Figure 13 shows the change of natural frequencies with the increasing of Young's modulus of cable material. It can be seen that all the natural frequencies of beam modes are nearly constant. But those of cable modes increase rapidly. Comparing the mode shapes at points $C$ and $D$ as shown in Figure 14, where the values of Young's modulus are $10 \mathrm{GPa}$ and $50 \mathrm{GPa}$, it can be seen that their natural frequencies almost have the same value. With the increase of Young's modulus, the deformation curve of the cable mode becomes more close to that of the beam mode.

\section{Conclusion}

In this paper, a Chebyshev spectral element method (CSEM) is presented for exploring the dynamic effects of cable attachment on a cantilever beam with tip mass. The cabled beam is modeled as a double-beam system connected by several springs at discrete locations. Using high order Chebyshev polynomials as basis functions, precise numerical results of system's natural frequencies and mode shapes can be obtained using fewer elements. This method divides the system according to the location of connections, not needing remeshing after the number of connections changed.

In addition, projection matrices are used to deal with differential boundary conditions. The results were compared with those of spectral element method in literature and those of finite element method based on software Nastran. It can be seen that the CSEM has a good convergence and accuracy.

Based on the validated CSEM, the effect of connection parameters, including their stiffness and number, was studied. The results show that the mode shapes can be classified
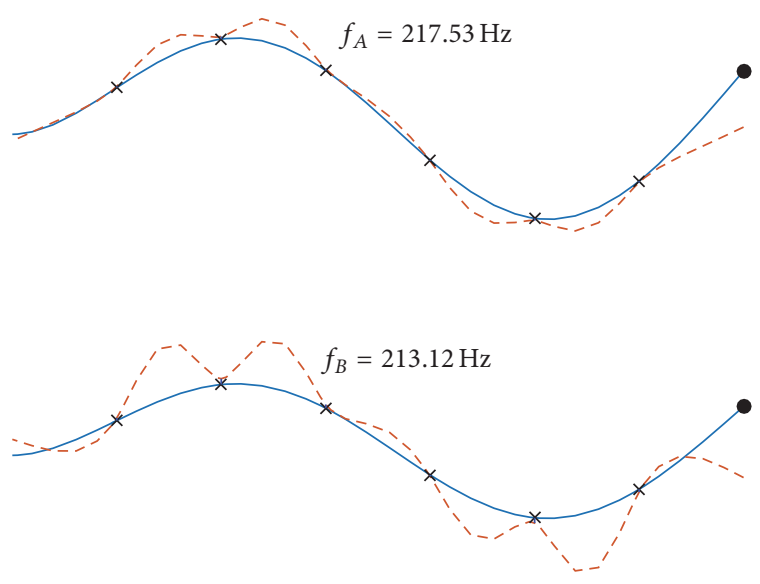

Figure 12: Mode shapes of $A, B$ points.

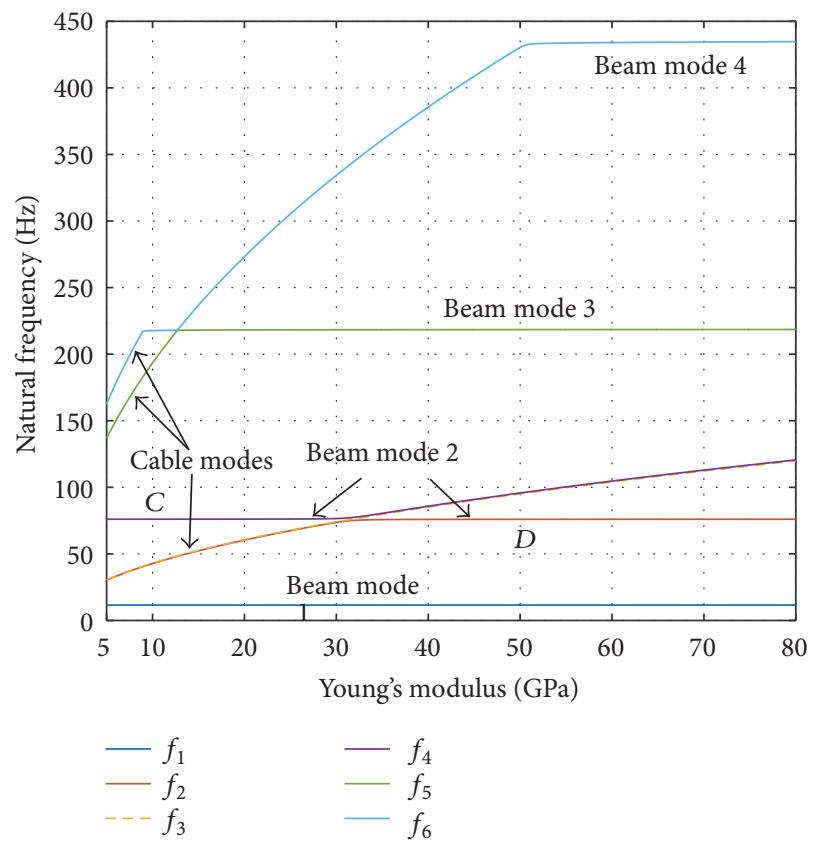

FIGURE 13: Variation of the natural frequencies with the cable's Young's modulus, 6 connections, and $k_{l}=10^{5} \mathrm{~N} / \mathrm{m}$.

into two types: beam mode shapes and cable mode shapes, according to their main deformation. With the increase of connection stiffness, the natural frequencies increase and finally converge to stable values. The low order frequencies increase and converge faster than the high order ones. The frequencies of beam modes increase and converge faster than those of cable modes. Changes of high order frequencies are much bigger than those of low order frequencies. As the number of connections increases, the natural frequencies of cable modes increase rapidly and the natural frequencies of beam modes keep nearly unchanged. The effects of cable's parameters, such as density and Young's modulus, are also investigated. It can be found that, with the increase of cable's density, the natural frequencies of cable modes decrease far more rapidly than those of beam modes. With the increase of Young's modulus of cable material, the natural frequencies of 

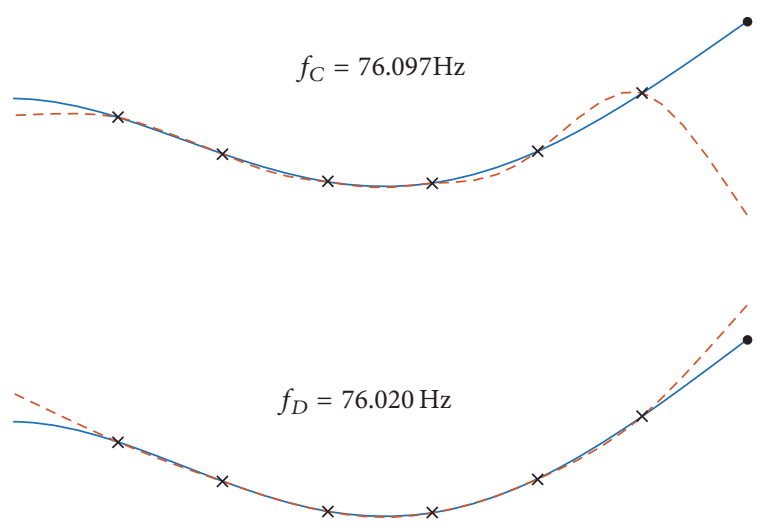

FIGURE 14: Mode shapes at points $C$ and $D$.

the beam modes keep nearly unchanged, and those of cable modes increase gradually. Meanwhile, the deformation curve of cable modes becomes more close to those of beam modes. These results can be applied to optimize the dynamic characteristics of precise cable-harnessed spacecraft structures.

\section{Competing Interests}

The authors declare that there is no conflict of interests regarding the publication of this paper.

\section{Acknowledgments}

This work is supported by the National Basic Research Program of China (Grant no. 2013CB733004).

\section{References}

[1] D. Coombs, R. Glaese, V. Babuška, L. Robertson, and B. Ingram, "Structural dynamic effects of cables on a sparse aperture deployable optical telescope," in Proceedings of the 49th AIAA/ ASME/ASCE/AHS/ASC Structures, Structural Dynamics, and Materials Conference, Schaumburg, Ill, USA, 2008.

[2] L. M. Robertson, S. A. Lane, B. R. Ingram et al., "Cable effects on the dynamics of large precision structures," in Proceedings of the 48th AIAA/ASME/ASCE/AHS/ASC Structures, Structural Dynamics, and Materials Conference, pp. 8236-8241, Honolulu, Hawaii, USA, April 2007.

[3] K. S. Spak, G. S. Agnes, and D. J. Inman, "Modeling vibration response and damping of cables and cabled structures," Journal of Sound \& Vibration, vol. 336, pp. 240-256, 2015.

[4] D. M. Coombs, J. C. Goodding, V. Babuška, E. V. Ardelean, L. M. Robertson, and S. A. Lane, "Dynamic modeling and experimental validation of a cable-loaded panel," Journal of Spacecraft and Rockets, vol. 48, no. 6, pp. 958-973, 2011.

[5] K. S. Spak, G. S. Agnes, and D. J. Inman, "Modeling vibration response and damping of cables and cabled structures," Journal of Sound and Vibration, vol. 336, pp. 240-256, 2015.

[6] J. Goodding, J. Griffee, and E. Ardelean, "Parameter estimation and structural dynamic modeling of electrical cable harnesses on precision structures," in Proceedings of the 49th AIAA/ ASME/ASCE/AHS/ASC Structures, Structural Dynamics, and Materials Conference, Schaumburg, Ill, USA, April 2008.
[7] J. Choi and D. J. Inman, "Development of modeling for cable harnessed structures," in Proceedings of the 54th AIAA/ASME/ ASCE/AHS/ASC Structures, Structural Dynamics and Materials Conference, Boston, Mass, USA, April 2013.

[8] G. Dai and W. Zhang, "Cell size effects for vibration analysis and design of sandwich beams," Acta Mechanica Sinica, vol. 25, no. 3, pp. 353-365, 2009.

[9] V. Stojanović and P. Kozić, Vibrations and Stability of Complex Beam Systems, Springer Tracts in Mechanical Engineering, Springer, 2015.

[10] Y. S. Hamed, M. Sayed, D.-X. Cao, and W. Zhang, "Nonlinear study of the dynamic behavior of a string-beam coupled system under combined excitations," Acta Mechanica Sinica, vol. 27, no. 6, pp. 1034-1051, 2011.

[11] V. Babuška, D. M. Coombs, J. C. Goodding, E. V. Ardelean, L. M. Robertson, and S. A. Lane, "Modeling and experimental validation of space structures with wiring harnesses," Journal of Spacecraft \& Rockets, vol. 47, no. 6, pp. 1038-1052, 2010.

[12] K. Spak, G. Agnes, and D. Inman, "Parameters for modeling stranded cables as structural beams," Experimental Mechanics, vol. 54, no. 9, pp. 1613-1626, 2014.

[13] K. Spak, G. Agnes, and D. Inman, "Comparison of damping models for space flight cables," in Topics in Dynamics of Civil Structures, vol. 4, Springer, New York, NY, USA, 2013.

[14] J. Choi and D. J. Inman, "Spectrally formulated modeling of a cable-harnessed structure," Journal of Sound and Vibration, no. 14, pp. 3286-3304, 2014.

[15] J. Choi, Investigation of the Dynamic Behavior of a CableHarnessed Structure, Virginia Tech, 2014.

[16] M. Remedia, G. S. Aglietti, and G. Richardson, "Modelling the effect of electrical harness on microvibration response of structures," Acta Astronautica, vol. 109, pp. 88-102, 2015.

[17] Z. Zhang, X. Huang, Z. Zhang, and H. Hua, "On the transverse vibration of Timoshenko double-beam systems coupled with various discontinuities," International Journal of Mechanical Sciences, vol. 89, pp. 222-241, 2014.

[18] B. Yagci, S. Filiz, L. L. Romero, and O. B. Ozdoganlar, "A spectral-Tchebychev technique for solving linear and nonlinear beam equations," Journal of Sound \& Vibration, vol. 321, no. 1-2, pp. 375-404, 2009.

[19] S. Filiz, L. A. Romero, and O. B. Ozdoganlar, "An analytical model for micro-endmill dynamics," Journal of Vibration \& Control, vol. 14, no. 8, pp. 1125-1150, 2008. 


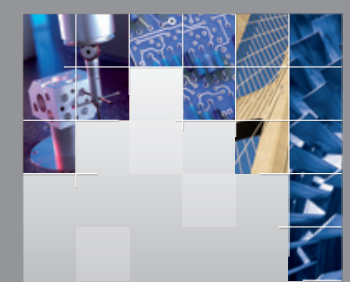

\section{Enfincering}
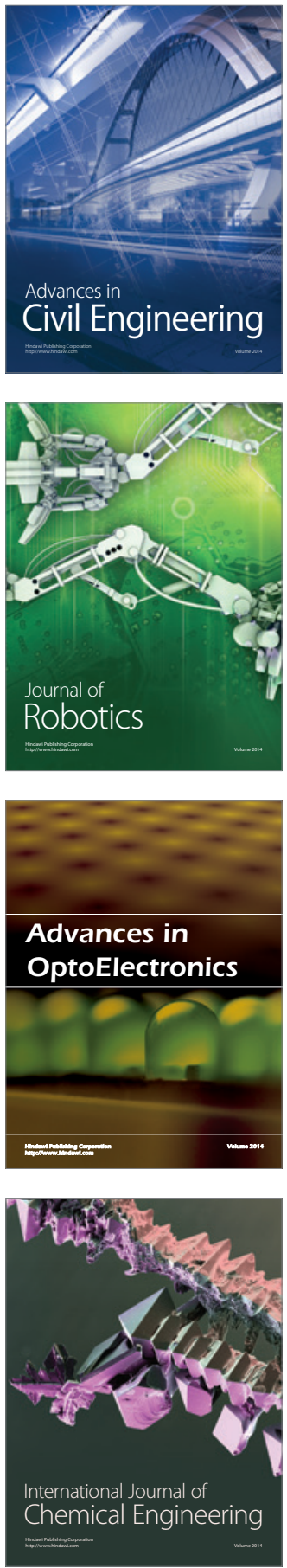

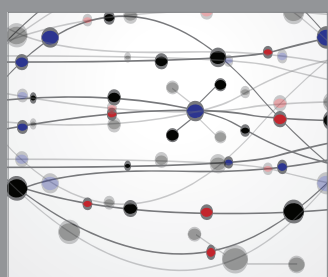

The Scientific World Journal

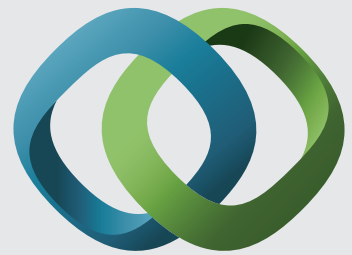

\section{Hindawi}

Submit your manuscripts at

http://www.hindawi.com
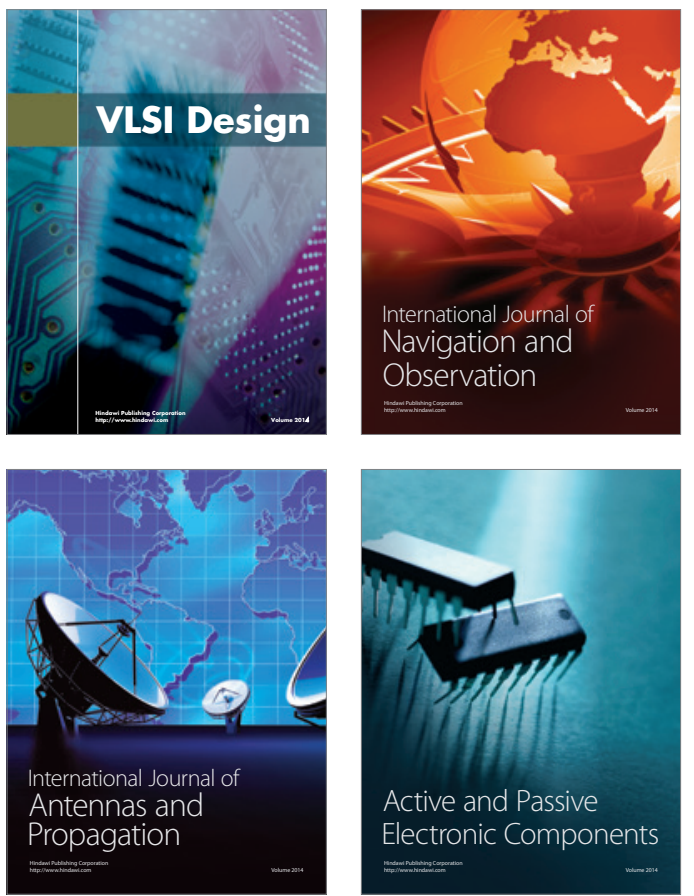
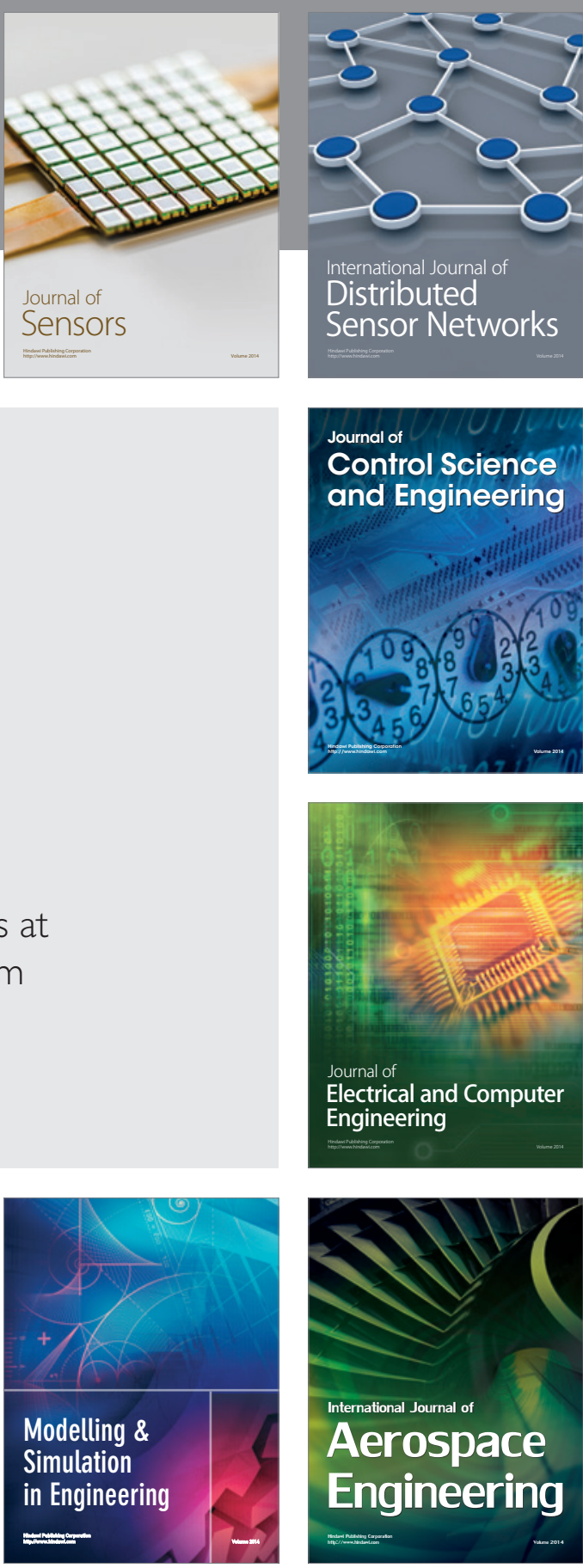

International Journal of

Distributed

Sensor Networks

Journal of

Control Science

and Engineering
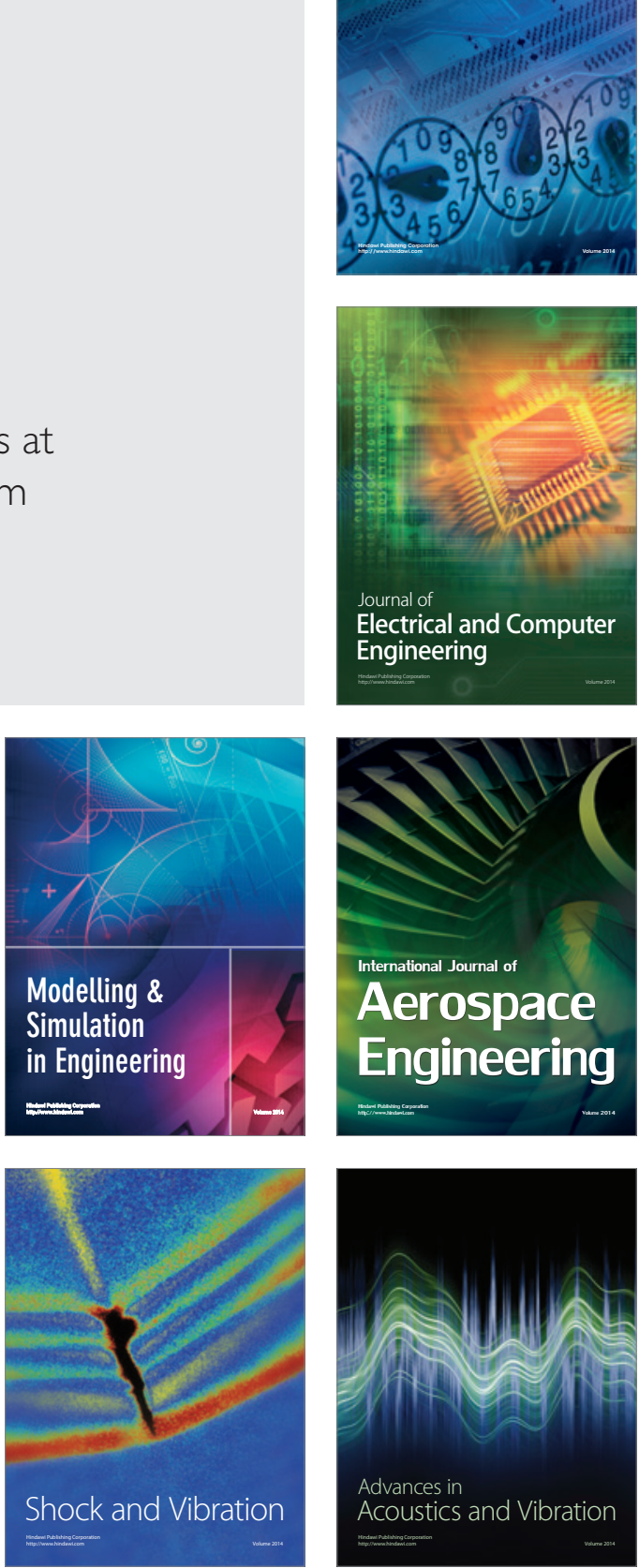